\title{
Twenty-Year Public Health Impact of 7- and 13-Valent Pneumococcal Conjugate Vaccines in US Children
}

\author{
Matt Wasserman, Ruth Chapman, Rotem Lapidot, Kelly Sutton, Desmond Dillon-Murphy, \\ Shreeya Patel, Erica Chilson, Vincenza Snow, Raymond Farkouh, Stephen Pelton
}

Pneumococcal conjugate vaccines (PCVs) have been used in the United States since 2000. To assess the cumulative 20-year effect of PCVs on invasive pneumococcal disease (IPD) incidence among children $<5$ years of age, we analyzed Active Bacterial Core Surveillance data, conducted a literature review, and modeled expected and observed disease. We found that PCVs have averted $>282,000$ cases of IPD, including $\approx 16,000$ meningitis, $\approx 172,000$ bacteremia, and $\approx 55,000$ bacteremic pneumonia cases. In addition, vaccination has prevented 97 million healthcare visits for otitis media, 438,914706,345 hospitalizations for pneumonia, and 2,780 total deaths. IPD cases declined 91\%, from 15,707 in 1997 to 1,382 in 2019 . Average annual visits for otitis media declined $41 \%$, from 78 visits/100 children before PCV introduction to 46 visits $/ 100$ children after PCV13 introduction. Annual pneumonia hospitalizations declined $66 \%-79 \%$, from $110,000-175,000$ in 1997 to 37,000 in 2019. These findings confirm the substantial benefits of PCVs for preventing IPD in children.

B efore 2000, children $<2$ years of age had the highest incidence of invasive pneumococcal diseases (IPDs) such as bacteremia, meningitis, or other infection of a normally sterile site (1). Researchers estimated that, in the United States, annual IPD incidence was 165 cases / 100,000 children $<12$ months of age and 203 cases /100,000 children 12-23 months of age (1). Until the United States began a universal 7-valent pneumococcal conjugate vaccine (PCV7) immunization program for children in 2000, Streptococcus pneumoniae was the leading cause of bacterial meningitis (1).

Author affiliations: Pfizer Inc., New York, New York, USA (M. Wasserman, E. Chilson, V. Snow, R. Farkouh); Evidera Market Access Ltd, London, UK (R. Chapman, K. Sutton, D. Dillon-Murphy, S. Patel); Boston University School of Medicine, Boston, Massachusetts, USA (R. Lapidot, S. Pelton)

DOI: https://doi.org/10.3201/eid2706.204238
S. pneumoniae was also the most common bacterial cause of community-acquired pneumonia and otitis media $(\mathrm{OM})$ in young children. Furthermore, in the 1990s, concerns emerged regarding the growing number of pneumococcal isolates with reduced susceptibility to first- and second-line antimicrobial drugs (1).

PCV7 was the first pneumococcal conjugate vaccine $(\mathrm{PCV})$ approved for use in children $<2$ years of age in the United States. Pneumococcal polysaccharide vaccines, which preceded PCVs, are not immunogenic in children $<2$ years of age $(1,2)$. PCV7 overcame the challenge of poor immunogenicity among infants and young children through conjugation technology; it was introduced into the US infant immunization schedule in 2000, providing direct protection against several serotypes of invasive and noninvasive pneumococcal disease $(3,4)$. PCV7 protects against the S. pneumoniae serotypes responsible for $>80 \%$ of IPD cases among children in North America (i.e., serotypes $4,6 \mathrm{~B}, 9 \mathrm{~V}, 14,18 \mathrm{C}, 19 \mathrm{~F}$, and $23 \mathrm{~F})(1,4)$. In 2010, PCV13, a vaccine providing protection against 6 additional serotypes (i.e., serotypes 1, 3, 5, 6A, 19A, and $7 \mathrm{~F}$ ), was approved in the United States, partially because of increasing incidence of serotypes not covered by PCV7 $(1,4)$.

Clinical trial data suggested that PCV7 would be effective against IPD, OM, and according to a posthoc analysis, pneumonia (5). The efficacy of PCV7 (and later PCV13) against all forms of pneumococcal disease was greater than expected, partly because of indirect protection gained through herd immunity $(6-8)$. The United States was the first country to introduce a PCV program for infants and, during the transition to PCV13, recommended the largest catchup program for children $<5$ years of age who had been vaccinated with PCV7 (9). After an initially slow uptake limited by constrained supply, the United States has achieved consistently high $(>80 \%)$ 3-dose 
coverage since 2005 (10). It is one of a few countries continuing to use the licensed 4-dose schedule (10). A 2020 review demonstrated that PCVs were the only vaccines approved by the US Food and Drug Administration that had no postmarketing safety-related label modifications (11).

We quantified the decrease of IPD incidence associated with 20 years of PCV use in the United States. First, we conducted a literature review to inform a decision analytic model. The model estimated the 20-year cumulative effects associated with the PCV program on cases of IPD, OM, and hospitalizations for pneumonia among children $<5$ years of age in the United States.

\section{Methods}

The US Centers for Disease Control and Prevention (CDC) began the Active Bacterial Core Surveillance (ABCs) program to monitor invasive $S$. pneumoniae infections in 1997 (12). Although this resource provides invaluable data for assessing IPD, it does not include data on noninvasive syndromes. We conducted a literature review to identify and synthesize published data on all pneumococcal diseases during the past 20 years. We used data from these publications to model the effects of PCVs on childhood pneumococcal disease (13).

\section{Literature Review}

To estimate the amount of pneumococcal disease averted in the United States, we conducted a systematic literature review in accordance with the Preferred Reporting Items for Systematic Reviews and MetaAnalyses guidelines (14). After defining the research questions, data sources, search strategies, and selection criteria (Appendix Tables 1-5, https://wwwnc.cdc. gov/EID/article/27/6/20-4238-App1.pdf), we conducted electronic searches of the PubMed and Embase (https://www.embase.com) databases and manual searches of the gray literature, CDC website (https:/ / www.cdc.gov), and reference lists of 7 published literature reviews (15-21) (Appendix Figure 1). This study describes only references used for input data or to validate our findings.

\section{Calculations and Outputs}

We developed a model using Excel (Microsoft, https://www.microsoft.com) to calculate the national numbers of cases, healthcare visits, hospitalizations, and deaths caused by pneumococcal infection among children $<5$ years of age during the 20 years after PCV introduction. We used published incidences of each syndrome (i.e., meningitis, bacteremia, bacteremic pneumonia/empyema, sepsis, and other) and relevant population data to calculate the number of cases averted by vaccination. We conducted these calculations for the pre-PCV (i.e., 1997-1999), PCV7 (i.e., 2000-2009), and PCV13 (i.e., 2010-2019) eras (Appendix Figure 2). Although we attributed decreasing illness and deaths to the direct effects of PCVs, policy changes or other interventions also might have contributed to the reduction of disease.

We calculated average incidences for each of the 3 described time periods. Because variance measures were unavailable, we performed all calculations as point estimates. We assumed that without PCVs, disease incidence would have remained constant. We calculated the estimated effect of PCV7 by comparing the difference in reported incidence between the pre-PCV and PCV7 eras; likewise, we considered the effect of PCV13 to be the difference in incidence between the pre-PCV and PCV13 eras. We estimated the incremental effect of including the additional serotypes in PCV13 by comparing incidence between the PCV7 and PCV13 eras. Because factors such as program rollout and uptake delayed the achievement of population-level equilibrium, we excluded the transition years 2000-2001 from the calculation of the effect of PCV7. Similarly, we excluded 2010 from the calculation of the effect of PCV13 (Tables 1, 2; Figures 1, 2). However, we included these years in the analysis of the 20-year aggregate effect of PCV use.

We calculated the number of expected IPD cases without PCV7 as the average incidence during 1997$1999 \times$ population size in each year. We calculated the expected IPD cases if PCV7 vaccination had continued but PCV13 had not been introduced as the average incidence during 2002-2009 $\times$ population size in each year. We stratified each calculation by age.

In addition, we calculated total IPD cases averted by PCVs as the difference between the cases expected without vaccination and the cases observed during 2002-2019 (Table 1; Appendix Figure 2). We also calculated the incremental effect of PCV13 versus PCV7 as the difference between cases expected if PCV7 use had continued after 2010 and cases observed during 2011-2019.

To calculate the number of expected IPD deaths without PCVs, we multiplied the observed case-fatality ratio from 1997-2000 (cumulative deaths divided by the cumulative cases in that period) by the expected number of cases from 2000-2019 (12). We considered deaths averted by PCVs to be the difference between expected deaths if PCVs had never been introduced and the observed deaths in this period. 
Case numbers and incidences were not available for $\mathrm{OM}$ and noninvasive pneumonia because they are nonnotifiable diseases. As a result, we calculated the expected ambulatory healthcare visit rates for $\mathrm{OM}$ and hospitalization rates for pneumonia without PCVs using the same method as for IPD cases averted.

\section{Model Inputs}

We conducted our calculations using population data from the US Census Bureau (22) (Appendix Table 6). We considered data on total IPD incidence, distri- bution of vaccine serotypes, syndrome distribution, healthcare visits for OM, and pneumonia incidence.

We obtained national estimates for IPD cases, rates, and syndromes among children $<1,1-<2$, and 2-4 years of age from ABCs reports (12) (Appendix Table 7). Because data for 2018 and 2019 were not available, we assumed these years to have the same rates as 2017. We used these data to calculate the average incidence for each of the 2 pre-PCV13 eras (Appendix Table 8).

We also obtained national estimates for overall annual incidence of IPD caused by PCV13 serotypes

\begin{tabular}{|c|c|c|c|c|c|c|c|c|c|c|}
\hline \multirow[b]{2}{*}{ Year } & \multicolumn{4}{|c|}{ No. observed, by age, y } & \multicolumn{4}{|c|}{ No. expected, by age, y } & \multicolumn{2}{|c|}{ No. averted } \\
\hline & $<1$ & 1 & $2-4$ & Overall & $<1$ & 1 & $2-4$ & Overall & Annual & Cumulative \\
\hline \multicolumn{11}{|c|}{ No. cases } \\
\hline 1997 & 5,360 & 6,712 & 3,635 & 15,707 & 5,360 & 6,712 & 3,635 & 15,707 & NA & NA \\
\hline 1998 & 6,220 & 7,630 & 4,286 & 18,136 & 6,220 & 7,630 & 4,286 & 18,136 & NA & NA \\
\hline 1999 & 6,176 & 7,772 & 3,837 & 17,785 & 6,176 & 7,772 & 3,837 & 17,785 & NA & NA \\
\hline 2000 & 5,699 & 6,139 & 3,469 & 15,306 & 6,053 & 7,428 & 3,883 & 17,364 & 2,057 & 2,057 \\
\hline 2001 & 2,099 & 2,645 & 3,143 & 7,887 & 6,299 & 7,539 & 3,852 & 17,689 & 9,802 & 11,860 \\
\hline 2002 & 1,521 & 1,261 & 1,813 & 4,596 & 6,202 & 7,830 & 3,866 & 17,899 & 13,304 & 25,164 \\
\hline 2003 & 1,642 & 1,405 & 1,530 & 4,577 & 6,241 & 7,696 & 3,936 & 17,874 & 13,296 & 38,460 \\
\hline 2004 & 1,485 & 1,253 & 1,454 & 4,192 & 6,301 & 7,730 & 3,983 & 18,013 & 13,821 & 52,281 \\
\hline 2005 & 1,450 & 1,419 & 1,467 & 4,336 & 6,286 & 7,796 & 4,019 & 18,101 & 13,765 & 66,046 \\
\hline 2006 & 1,366 & 1,458 & 1,395 & 4,219 & 6,344 & 7,767 & 4,019 & 18,130 & 13,911 & 79,956 \\
\hline 2007 & 1,680 & 1,296 & 1,560 & 4,537 & 6,511 & 7,826 & 4,036 & 18,372 & 13,835 & 93,792 \\
\hline 2008 & 1,517 & 1,284 & 1,420 & 4,221 & 6,487 & 8,018 & 4,057 & 18,562 & 14,341 & 108,133 \\
\hline 2009 & 1,485 & 1,309 & 1,654 & 4,449 & 6,284 & 7,975 & 4,099 & 18,358 & 13,910 & 122,043 \\
\hline 2010 & 1,352 & 1,051 & 1,611 & 4,014 & 6,204 & 7,726 & 4,144 & 18,074 & 14,060 & 136,103 \\
\hline 2011 & 832 & 670 & 1,012 & 2,515 & 6,221 & 7,755 & 4,109 & 18,085 & 15,571 & 151,674 \\
\hline 2012 & 616 & 541 & 712 & 1,870 & 6,163 & 7,777 & 4,068 & 18,009 & 16,139 & 167,813 \\
\hline 2013 & 578 & 595 & 814 & 1,988 & 6,171 & 7,709 & 4,036 & 17,916 & 15,928 & 183,741 \\
\hline 2014 & 629 & 407 & 754 & 1,790 & 6,208 & 7,721 & 4,033 & 17,963 & 16,173 & 199,914 \\
\hline 2015 & 733 & 513 & 610 & 1,856 & 6,253 & 7,769 & 4,031 & 18,053 & 16,198 & 216,112 \\
\hline 2016 & 526 & 212 & 167 & 906 & 6,208 & 7,828 & 4,032 & 18,068 & 17,163 & 233,275 \\
\hline 2017 & 452 & 314 & 625 & 1,391 & 6,112 & 7,770 & 4,052 & 17,934 & 16,544 & 249,818 \\
\hline 2018 & 446 & 309 & 627 & 1,382 & 6,040 & 7,651 & 4,061 & 17,752 & 16,370 & 266,188 \\
\hline 2019 & 446 & 309 & 627 & 1,382 & 6,040 & 7,651 & 4,061 & 17,752 & 16,370 & 282,558 \\
\hline \multicolumn{11}{|c|}{ No. deaths } \\
\hline 1997 & 151 & 34 & 17 & 202 & 151 & 34 & 17 & 202 & NA & NA \\
\hline 1998 & 78 & 47 & NA & 126 & 78 & 47 & NA & 126 & NA & NA \\
\hline 1999 & 29 & 45 & 60 & 134 & 29 & 45 & 60 & 134 & NA & NA \\
\hline 2000 & 130 & 113 & 57 & 301 & 138 & 137 & 64 & 340 & 39 & 39 \\
\hline 2001 & 22 & 46 & 57 & 125 & 103 & 66 & 36 & 204 & 79 & 118 \\
\hline 2002 & 31 & NA & 32 & 62 & 101 & 68 & 36 & 205 & 143 & 261 \\
\hline 2003 & 42 & 21 & 21 & 85 & 102 & 67 & 37 & 206 & 121 & 382 \\
\hline 2004 & 39 & 48 & 20 & 107 & 103 & 67 & 37 & 207 & 101 & 483 \\
\hline 2005 & 38 & 9 & 38 & 85 & 103 & 68 & 37 & 208 & 123 & 605 \\
\hline 2006 & 29 & 9 & 47 & 85 & 104 & 68 & 37 & 209 & 124 & 729 \\
\hline 2007 & 37 & 9 & 9 & 56 & 106 & 68 & 37 & 212 & 156 & 885 \\
\hline 2008 & 37 & NA & 18 & 55 & 106 & 70 & 38 & 214 & 158 & 1,043 \\
\hline 2009 & 36 & 9 & 9 & 54 & 103 & 70 & 38 & 210 & 156 & 1,199 \\
\hline 2010 & 10 & 9 & 9 & 28 & 101 & 67 & 38 & 207 & 179 & 1,378 \\
\hline 2011 & 38 & 19 & 28 & 85 & 102 & 68 & 38 & 207 & 123 & 1,501 \\
\hline 2012 & 9 & 9 & NA & 19 & 101 & 68 & 38 & 206 & 187 & 1,688 \\
\hline 2013 & 9 & 9 & 38 & 57 & 101 & 67 & 37 & 205 & 149 & 1,837 \\
\hline 2014 & 19 & NA & 9 & 28 & 101 & 67 & 37 & 206 & 178 & 2,014 \\
\hline 2015 & 10 & 9 & 19 & 38 & 102 & 68 & 37 & 207 & 170 & 2,184 \\
\hline 2016 & 18 & 4 & 12 & 35 & 101 & 68 & 37 & 207 & 172 & 2,356 \\
\hline 2017 & 28 & 19 & 38 & 85 & 100 & 68 & 38 & 205 & 120 & 2,476 \\
\hline 2018 & 16 & 7 & 28 & 51 & 99 & 67 & 38 & 203 & 152 & 2,628 \\
\hline 2019 & 16 & 7 & 28 & 51 & 99 & 67 & 38 & 203 & 152 & 2,780 \\
\hline
\end{tabular}

*Values are rounded to the nearest whole numbers. NA, not applicable. 
Table 2. Cases of invasive pneumococcal disease averted by PCV13, United States, 1997-2019*

\begin{tabular}{|c|c|c|c|c|c|c|c|c|c|}
\hline \multirow[b]{2}{*}{ Year } & \multicolumn{3}{|c|}{ No. observed } & \multicolumn{3}{|c|}{ No. expected } & \multicolumn{2}{|c|}{ Cumulative cases averted } & \multirow{2}{*}{$\begin{array}{c}\text { Difference in } \\
\text { non-PCV13 } \\
\text { serotype cases }\end{array}$} \\
\hline & All & $\begin{array}{l}\text { PCV13 } \\
\text { serotypes }\end{array}$ & $\begin{array}{l}\text { Non-PCV13 } \\
\text { serotypes }\end{array}$ & All & $\begin{array}{l}\text { PCV13 } \\
\text { serotypes }\end{array}$ & $\begin{array}{l}\text { Non-PCV13 } \\
\text { serotypes }\end{array}$ & All & $\begin{array}{l}\text { PCV13 } \\
\text { serotypes }\end{array}$ & \\
\hline 1997 & 15,543 & 14,439 & 1,104 & 15,543 & 14,439 & 1,104 & NA & NA & NA \\
\hline 1998 & 18,136 & 16,848 & 1,288 & 18,136 & 16,848 & 1,288 & NA & NA & NA \\
\hline 1999 & 17,785 & 16,839 & 945 & 17,785 & 16,839 & 945 & NA & NA & NA \\
\hline 2000 & 15,306 & 13,808 & 1,498 & 17,364 & 16,178 & 1,186 & 2,057 & 2,369 & -312 \\
\hline 2001 & 7,887 & 6,561 & 1,325 & 17,689 & 16,481 & 1,208 & 11,860 & 12,289 & -429 \\
\hline 2002 & 4,596 & 3,109 & 1,487 & 17,899 & 16,677 & 1,223 & 25,164 & 25,857 & -693 \\
\hline 2003 & 4,577 & 2,743 & 1,834 & 17,874 & 16,653 & 1,221 & 38,460 & 39,767 & $-1,307$ \\
\hline 2004 & 4,192 & 2,374 & 1,818 & 18,013 & 16,783 & 1,230 & 52,281 & 54,175 & $-1,894$ \\
\hline 2005 & 4,336 & 2,589 & 1,747 & 18,101 & 16,864 & 1,236 & 66,046 & 68,450 & $-2,405$ \\
\hline 2006 & 4,219 & 2,592 & 1,627 & 18,130 & 16,891 & 1,238 & 79,956 & 82,750 & $-2,793$ \\
\hline 2007 & 4,537 & 3,019 & 1,518 & 18,372 & 17,118 & 1,255 & 93,792 & 96,848 & $-3,057$ \\
\hline 2008 & 4,221 & 2,635 & 1,585 & 18,562 & 17,294 & 1,268 & 108,133 & 111,507 & $-3,374$ \\
\hline 2009 & 4,449 & 3,037 & 1,412 & 18,358 & 17,104 & 1,254 & 122,043 & 125,575 & $-3,532$ \\
\hline 2010 & 4,014 & 2,626 & 1,388 & 18,074 & 16,839 & 1,235 & 136,103 & 139,788 & $-3,685$ \\
\hline 2011 & 2,515 & 805 & 1,710 & 18,085 & 16,850 & 1,235 & 151,674 & 155,833 & $-4,160$ \\
\hline 2012 & 1,870 & 400 & 1,470 & 18,009 & 16,779 & 1,230 & 167,813 & 172,213 & $-4,400$ \\
\hline 2013 & 1,988 & 397 & 1,591 & 17,916 & 16,692 & 1,224 & 183,741 & 188,508 & $-4,766$ \\
\hline 2014 & 1,790 & 397 & 1,392 & 17,963 & 16,736 & 1,227 & 199,914 & 204,846 & $-4,932$ \\
\hline 2015 & 1,856 & 398 & 1,457 & 18,053 & 16,820 & 1,233 & 216,112 & 221,268 & $-5,156$ \\
\hline 2016 & 906 & 398 & 507 & 18,068 & 16,834 & 1,234 & 233,275 & 237,703 & $-4,429$ \\
\hline 2017 & 1,391 & 398 & 993 & 17,934 & 16,709 & 1,225 & 249,818 & 254,015 & $-4,197$ \\
\hline 2018 & 1,382 & 396 & 986 & 17,752 & 16,539 & 1,213 & 266,188 & 270,158 & $-3,970$ \\
\hline 2019 & 1,382 & 396 & 986 & 17,752 & 16,539 & 1,213 & 282,558 & 286,302 & $-3,744$ \\
\hline
\end{tabular}

*Values are rounded to the nearest whole numbers. NA, not applicable; PCV13, 13-valent pneumococcal conjugate vaccine.

†Negative values indicate greater number observed than would be expected.

among children < 5 years of age during 1998-2016 (12) (Appendix Table 9). We assumed rates during 2017-2019 to be the same as 2016; we weighted these rates by population distribution during those years. We calculated pre-PCV era distribution of PCV13 and non-PCV13 serotypes as the average of 19971999 distributions (Appendix Table 10). To ensure serotype incidences were consistent with the observed trends of all IPDs, we imputed PCV13 serotype incidence in 1997 using weighted proportions (i.e., by population size in each age group) and percent change (Appendix Table 9) during 1997-1998. We calculated expected cases caused by PCV13 and non-PCV13 serotypes by multiplying the average pre-PCV era serotype distributions to the total expected number of annual IPD cases. Although the measurement of all averted cases of IPD includes the effects of vaccination and serotype replacement, the measurement of cases averted by PCV13 indicates only the reduction in vaccine type IPDs.

We obtained the proportions of meningitis, bacteremia, bacteremic pneumonia/empyema, sepsis, and other infections among children $<2$ and $2-4$ years of age with IPD from additional unpublished data provided by CDC (12; R. Gierke, CDC, pers. comm., 2017 Nov 7) (Appendix Table 11). We assumed the distributions in 1997-1999 to be the same as 2000 .

We used the mean rate of ambulatory care visits for $\mathrm{OM}$ in children $<2,2-<5$, and $<5$ years of age overall provided by Zhou et al. (23; Appendix Table 12, Figure 3). Zhou et al. (23) described PCV eras using similar definitions: the pre-PCV period during 1997-1998, the PCV7 period during 2002-2009, and the PCV13 period during 2011-2013 (Appendix Table 13). We assumed the rates in 2014-2019 to be the same as 2013 (Appendix Table 12).

The data sources used various classifications and definitions of pneumonia. The types of data reported also varied widely, including measurements such as ambulatory visits, hospitalizations, index cases in inpatients, and estimates of cases of community-acquired pneumonia. No single data source covered the combined PCV7 and PCV13 periods, nor estimated the incidence of only noninvasive pneumococcal pneumonia. Because hospitalization data represent more severe cases with the largest use of healthcare costs and resources and because no consistent data for ambulatory/outpatient visits for pneumonia during the entire study period were available, we considered only hospitalized cases of pneumonia in this analysis. We used data on hospitalization for pneumonia from multiple sources. We obtained data for the pre-PCV relative to $\mathrm{PCV} 7$ eras from Simonsen et al. (24), Foote et al. (25), and Grijalva et al. (26), and for the PCV7 relative to PCV13 period from a 2005-2014 study (27) and Tong et al. (28). In addition, we used estimates of the difference in hospitalization incidences during the PCV7 
period from Grijalva et al. (23). We estimated the total number of hospitalizations averted by PCVs using the expected hospitalization data from all sources for 1997-2019 (Appendix Table 14).

\section{Validation}

During the literature review, we identified appropriate references against which to validate the consistency of our findings. We did not identify other sources of national multistate data for IPD comparable to the ABCs dataset. Black et al. (30) reported Kaiser Permanente data from northern California about the effect of PCV7 on disease epidemiology in children and adults, whereas Yildirim et al. (31) reported serotype-specific invasive capacity among children in Massachusetts after PCV introduction (Appendix Figure 4).

We used a large national claims database (32), a commercial claims and encounters database (33), and Ray et al. (34) to validate our OM estimates. We wanted to validate our pneumonia estimates with respect to different types of cases and definitions used by various data sources; however, because of constraints on data availability, we limited our validation to hospitalized cases of all-cause pneumonia. Because of the variation in reporting of pneumonia data, we did not identify any alternate sources for an appropriate validation of our analysis.

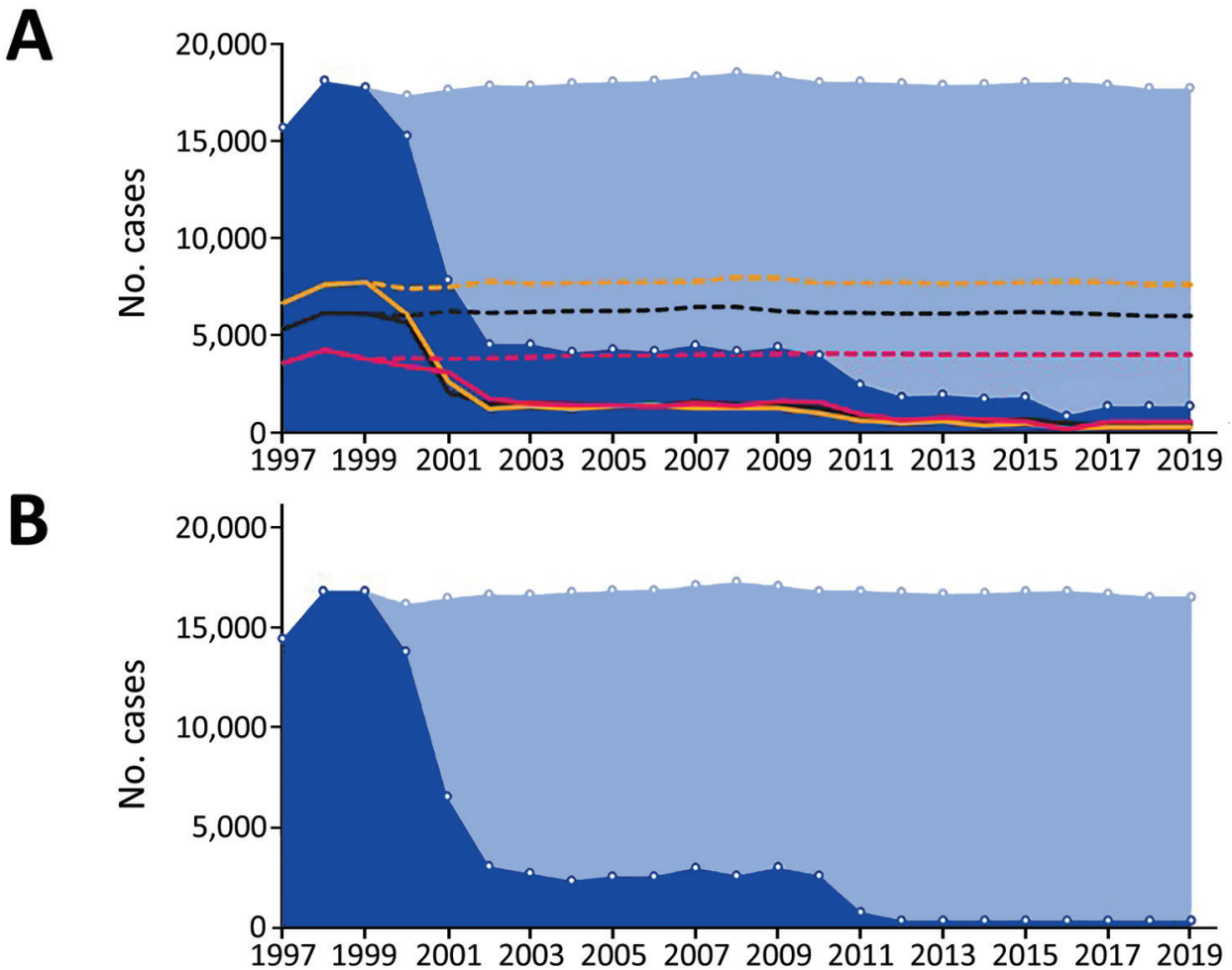

Figure 1. Effects of PCVs on invasive pneumococcal disease (IPD) and otitis media among children $<5$ years of age, United States, 1997-2019 (8,12). A) Cases of IPD. B) Cases of IPD caused by 13-valent PCV serotypes. C) Healthcare visits for otitis media. The United States approved 7-valent PCV in 2000 and 13-valent PCV in 2010. Asterisk $\left(^{*}\right)$ indicates that for data on healthcare visits for otitis media, age range is $0-2$ years. PCV, pneumococcal conjugate vaccine.

\section{C}

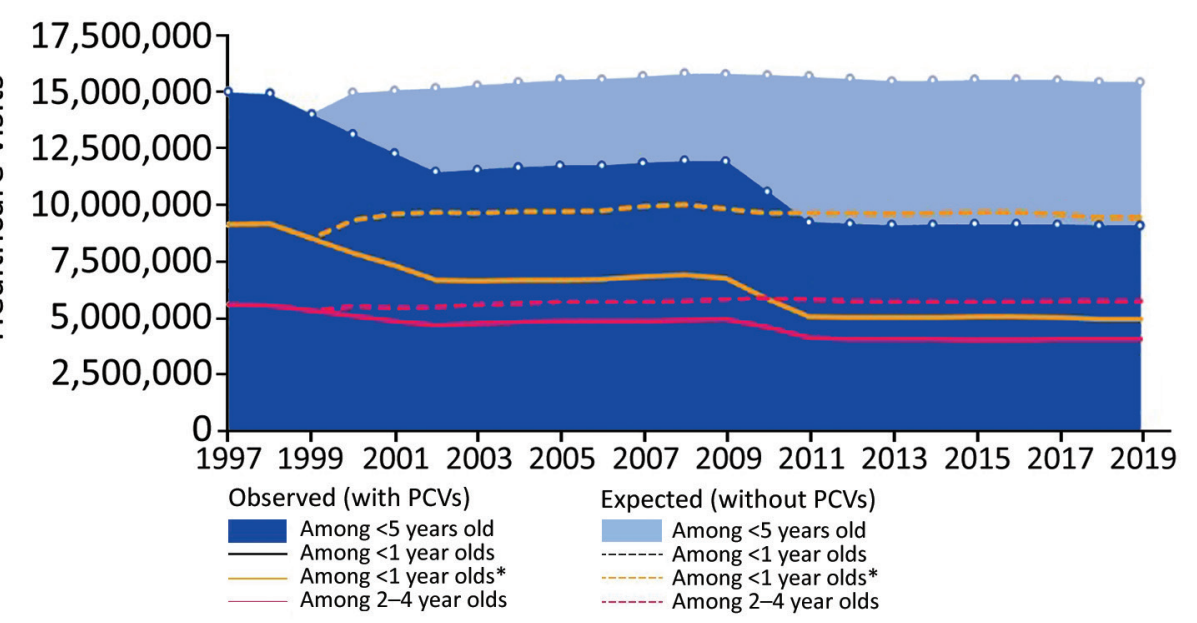




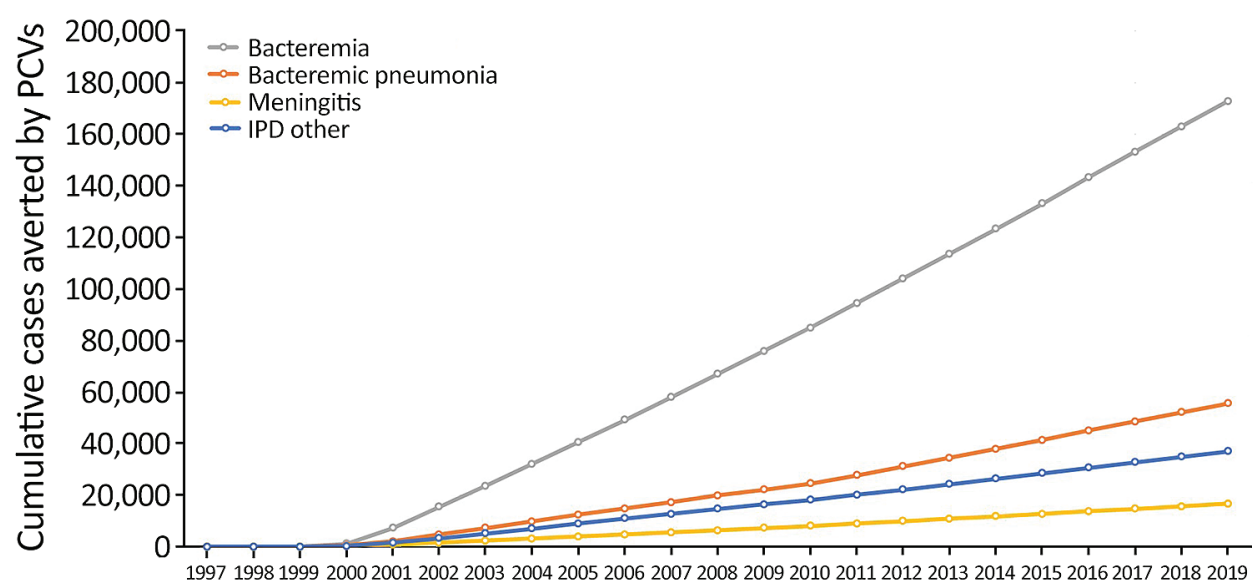

Figure 2. Effects of PCVs on different syndromes of IPD in children $<5$ years of age, United States, 1997-2019. The United States approved 7-valent PCV in 2000 and 13-valent PCV in 2010. IPD, invasive pneumococcal disease; PCV, pneumococcal conjugate vaccine.

\section{Results}

\section{IPD}

Among children $<5$ years of age, the annual number of IPD cases decreased from $\approx 16,000-18,000$ during 1997-1999 to 1,382 in 2019 (Table 1; Figure 1, panel A). We estimated that PCVs averted a cumulative 282,558 cases of IPD during this timeframe. Of those averted cases, we estimated that 146,455 were prevented by PCV13 during 2010-2019. Among children <5 years of age, annual deaths caused by IPD decreased from 126-202 during 1997-1999 (Table 1) to 85 in 2019. We estimated that PCVs prevented a total of 2,780 deaths during this timeframe, including 1,402 deaths prevented by PCV13 during 2010-2019.

The overall IPD incidences in the input ABCs data were generally higher than in the sources used for validation. However, the ABCs and the Kaiser Permanente data (30) reflected similar overall trends for the pre-PCV and PCV7 eras; the ABCs and data from Yildrim et al. (31) reflected similar overall trends for the PCV7 and PCV13 eras. The differences were probably caused by variations in reporting and patient groups between data sources; the ABCs data are more nationally representative and therefore more generalizable than the population described by Black et al. (30).

We observed a decrease in IPD-related deaths after the introduction of PCV7; however, we could not identify whether this trend existed during the prePCV period because of limited data (Table 1). Pulido et al. (35) described a declining IPD mortality rate during 1990-2005, supporting the ABCs data and indicating that deaths were already decreasing before the introduction of PCVs. Reductions in smoking rates and implementation of laws regarding smoking in public places, shifts from inpatient to outpatient care settings, improved treatments, and varying case definitions might have also contributed to the declining trend.

We estimated that during 2000-2019, PCVs prevented 172,778 cases of bacteremia; 55,532 cases of bacteremic pneumonia and empyema; 16,660 cases of meningitis; and 37,017 cases of other forms of IPD (Figure 2). IPD cases caused by PCV13 serotypes decreased from 14,439 cases in 1997 to 396 in 2019 (Table 2; Figure 1, panel B). During 2000-2019, PCVs are estimated to have averted 286,302 IPD cases caused by vaccine serotypes. During this period, IPD cases caused by non-PCV13 serotypes increased slightly, consistent with modest serotype replacement.

\section{OM Healthcare Visits}

The average rate of $\mathrm{OM}$ visits among children $<5$ years of age declined from 78/100 to 46/100 children per year from the pre-PCV (1997-1998) to the PCV13 era (2011-2013). In other words, these visits declined by $39 \%$, from $15,000,483$ in 1997 to $9,112,727$ in 2019

\begin{tabular}{|c|c|c|c|c|}
\hline Measure & 1997-1999 & $2000-2009$ & 2010-2019 & Cumulative \\
\hline Average incidence of visits per 100 children & 78 & 59 & 46 & NA \\
\hline Expected visits $†$ & NA & $154,269,900$ & $155,511,917$ & $309,781,817$ \\
\hline Estimated visits & NA & $119,429,938$ & $93,025,190$ & $212,455,128$ \\
\hline Visits averted $\ddagger$ & NA & $34,839,962$ & $62,486,726$ & $97,326,688$ \\
\hline
\end{tabular}


Table 4. Estimated total hospitalized cases of pneumonia averted by PCVs, United States, 1997-2019*

\begin{tabular}{|c|c|c|c|c|c|c|}
\hline \multirow[b]{3}{*}{ Time period } & \multicolumn{4}{|c|}{ Estimated hospitalizations } & \multirow{2}{*}{\multicolumn{2}{|c|}{ Total hospitalizations averted }} \\
\hline & \multicolumn{2}{|c|}{ Observed, with vaccination } & \multicolumn{2}{|c|}{ Expected without vaccination } & & \\
\hline & Minimum & Maximum & Minimum & Maximum & Minimum & Maximum \\
\hline Pre-PCV era: 1997-1999 & NA & NA & 339,474 & 525,675 & NA & NA \\
\hline PCV7 era: $2000-2009 \dagger$ & 959,543 & $1,336,673$ & 597,479 & $1,822,591$ & 222,611 & $490,043 \ddagger$ \\
\hline PCV13 era: $2010-2019 \S$ & \multicolumn{2}{|c|}{382,182} & \multicolumn{2}{|c|}{598,484} & \multicolumn{2}{|c|}{216,303} \\
\hline $\begin{array}{l}\text { *PCV7 was approved for use } \\
\text { pneumococcal conjugate vac } \\
\dagger \text { Averted hospitalizations dur } \\
\text { based on Grijalva et al (29) } \\
\text { †Grijalva et al (29) reported } \\
\text { §Values based on calculation }\end{array}$ & lest $\mathrm{PC}$ & $\begin{array}{l}\text { V13 was a } \\
13,13 \text {-vale } \\
\text { difference } \\
\text { c.cdc.gov/ }\end{array}$ & or use in th & $\begin{array}{l}\text { States in } 2 \\
\text { imum bas }\end{array}$ & $\begin{array}{l}\text { A, not al } \\
\text { monsor }\end{array}$ & $\mathrm{CV}$ \\
\hline
\end{tabular}

(Figure 1, panel C). We estimated that PCVs averted a cumulative 97,326,688 OM-related healthcare visits (Table 3).

The overall visit numbers in the input data (23) were generally lower than in the sources used for validation (32-34), but the overall trends for the prePCV and PCV7 eras were comparable. The differences might have been caused by varying database populations, because Zhou et al. (23) used national data whereas Marom et al. (32) and Tong et al. (33) mainly considered privately insured patients who might have been more likely to seek care. Therefore, the estimates from Zhou et al. (23) are probably more representative on a national level.

\section{Pneumonia Hospitalizations}

Annual pneumonia hospitalizations in children $<5$ years of age declined from 113,116-175,420 in 1997 to 37,882 in 2019. We estimated that PCVs averted a cumulative 438,914-706,345 pneumonia hospitalizations, including 216,303 cases caused by PCV13 serotypes, during the 20 years after PCV introduction in the United States (Table 4).

\section{Discussion}

Vaccines, especially PCVs, are lifesaving and costeffective public health interventions. At the time PCV7 was introduced in the United States, pneumococcal disease caused high rates of death and disease among infants. Despite conservative findings from the clinical trials (8), public health officials and healthcare professionals were optimistic about the potential of PCVs to prevent pneumococcal disease. We conducted a literature review and modeling analysis to quantify the effects of PCVs on pneumococcal disease incidence among children $<5$ years of age, a population at higher risk for IPD and therefore the focus of IPD prevention efforts (36). Our analysis demonstrated that PCVs have averted $>282,000$ cases of IPD and 2,780 associated deaths, with reductions across various IPD syndromes. Since their introduction in 2000, PCVs have averted $>430,000$ pneumonia hospitalizations and >97,000,000 OMrelated healthcare visits.

We could not find data on all OM cases; our analysis instead measured ambulatory care visits using input data from Zhou et al. (23). However, because not all children with OM receive treatment through ambulatory care visits, our findings probably underestimate the true effects of PCVs on OM incidence. The annual number of $\mathrm{OM}$ visits declined from 78 visits / 100 to 46 visits/100 children from the pre-PCV era (1997-1999) to PCV13 era (2010-2019); this $41 \%$ decline exceeds the original predictions based on early clinical trial data (8). Vaccination is probably the main direct contributor to this reduction; however, vaccination also might have had indirect effects such as changes in the disease definition or clinical coding of OM, as well as changes in prescribing patterns of antimicrobial drugs, which might affect healthcare use. Although not all cases of OM prompt healthcare visits, even mild illnesses might require family members to take time from work to care for their children, further reducing productivity and quality of life in ways not reflected by this metric (37).

Because we could not find data on noninvasive pneumonia, we combined multiple data sources to compare pneumonia hospitalizations during the study period. These data are probably underestimates of the true effect of PCVs because most pneumonia cases among children $<5$ years of age do not require hospitalization.

The overall findings are impressive but nevertheless conservative. We did not consider the direct benefits of reduced sequelae among children $>5$ years of age nor adults; we also did not consider indirect benefits such as herd immunity, reduced use of antimicrobial drugs and other healthcare resources, increased educational attainment, or improved parental productivity. In addition, we did not analyze data on common but less resource-intensive manifestations of S. pneumoniae such as conjunctivitis $(38,39)$. Finally, this analysis does not reflect PCVs' effects on antimicrobial resistance 
(17), although preventing infection through vaccination reduces the need for antimicrobial treatment (40).

In agreement with other studies $(40,41)$, we found that IPD cases caused by PCV13 serotypes declined while the number of cases caused by nonPCV13 serotypes slightly increased, reflecting modest serotype replacement. PCVs have been used in the United States longer than in any other country. Because of the large quantity of available data, we conducted a literature review to independently identify appropriate references for model inputs and validation. However, although the available data were extensive, it was not comprehensive. As a result, this research was limited by the lack of a single data source for pneumonia and OM incidences during the entire study period, prompting us to impute values for years when no data were available. In addition, because IPD is a notifiable disease but $\mathrm{OM}$ and pneumonia are not, we can only estimate PCVs' true effects on OM and pneumonia incidence using alternative metrics such as ambulatory care visits and hospitalizations. Furthermore, healthcare providers do not usually distinguish the causative bacteria of pneumonia and OM cases, which poses difficulties in analyzing serotype distributions. Finally, we could not find alternative national-level IPD data for the validation analysis, prompting us to compare our results with trends from smaller regions.

CDC and the Advisory Committee on Immunization Practices have recommended the use of PCVs in a national infant immunization program since 2000 (1). Our model used available data to quantify the effects of PCV7 and PCV13 on pneumococcal disease burden among children in the United States. Our results demonstrate the effectiveness of PCVs in preventing illness and death among children $<5$ years of age.

\section{About the Author}

Mr. Wasserman is Director of Health Economics and Outcomes Research at Pfizer Inc. His research interests include cost-effectiveness and transmission dynamic modeling of vaccine-preventable diseases.

This work was supported by Pfizer Inc. Pfizer participated in study design; data identification, synthesis, and analysis; manuscript writing; and the decision to submit the article for publication.

E.C., V.S., R.F., and M.W. are employees and stockholders of Pfizer Inc. R.C., K.S., D.D.M., and S.P. are employees of Evidera Market Access Ltd, a healthcare research firm that provides consulting and other research services to pharmaceutical and related organizations. In these salaried positions, they work with a variety of companies and are explicitly precluded from accepting any payment or honoraria directly from them for services rendered. Evidera received payment from Pfizer Inc. for collaboration on this project and article.

S.P. and R.L. received consulting fees from Pfizer, Inc. for their participation in study design and data analysis for this project. S.P. and R.L. are also recipients of investigator-initiated research awards (i.e., Surveillance of Pneumococcal Colonization and Invasive pneumococcal Disease reveals Shift in Prevalent Carriage Serotypes in Massachusetts' Children to Relatively Low Invasiveness [31], Evaluation of Serotype 3 Strains collected from Massachusetts' Children 2002-2019) from Pfizer (through Boston Medical Center) relevant to pneumococcal vaccine studies. S.P. has also received honoraria from Merck, Pfizer, and Sanofi for participation in pneumococcal advisory boards and Data and Safety Monitoring Board.

\section{References}

1. Advisory Committee on Immunization Practices. Preventing pneumococcal disease among infants and young children. Recommendations of the Advisory Committee on Immunization Practices (ACIP). MMWR Recomm Rep. 2000;49:1-35.

2. Douglas RM, Paton JC, Duncan SJ, Hansman DJ. Antibody response to pneumococcal vaccination in children younger than five years of age. J Infect Dis. 1983;148:131-7. https://doi.org/10.1093/infdis/148.1.131

3. Centers for Disease Control and Prevention. Direct and indirect effects of routine vaccination of children with 7 -valent pneumococcal conjugate vaccine on incidence of invasive pneumococcal disease - United States, 1998-2003. MMWR Morb Mortal Wkly Rep. 2005;54:893-7.

4. Kellner JD, Vanderkooi OG, MacDonald J, Church DL, Tyrrell GJ, Scheifele DW. Changing epidemiology of invasive pneumococcal disease in Canada, 1998-2007: update from the Calgary-area Streptococcus pneumoniae research (CASPER) study. Clin Infect Dis. 2009;49:205-12. https:/ / doi.org/10.1086/599827

5. Black S, Shinefield H, Fireman B, Lewis E, Ray P, Hansen JR,et al.; Northern California Kaiser Permanente Vaccine Study Center Group. Efficacy, safety and immunogenicity of heptavalent pneumococcal conjugate vaccine in children. Pediatr Infect Dis J. 2000;19:187-95. https://doi.org/10.1097/00006454-200003000-00003

6. Haber M, Barskey A, Baughman W, Barker L, Whitney CG, Shaw KM, et al. Herd immunity and pneumococcal conjugate vaccine: a quantitative model. Vaccine. 2007; 25:5390-8. https://doi.org/10.1016/j.vaccine.2007.04.088

7. Miller E, Andrews NJ, Waight PA, Slack MP, George RC. Herd immunity and serotype replacement 4 years after seven-valent pneumococcal conjugate vaccination in England and Wales: an observational cohort study. Lancet Infect Dis. 2011;11:760-8. https:/ / doi.org/10.1016/ S1473-3099(11)70090-1

8. Eskola J. Polysaccharide-based pneumococcal vaccines in the prevention of acute otitis media. Vaccine. 2000;19:S78-82. https://doi.org/10.1016/S0264-410X(00)00283-8 
9. Strutton DR, Farkouh RA, Rubin JL, McGarry LJ, Loiacono PM, Klugman KP, et al. Modeling the impact of the 13-valent pneumococcal conjugate vaccine serotype catch-up program using United States claims data. BMC Infect Dis. 2012;12:175. https:/ / doi.org/10.1186/1471-2334-12-175

10. Centers for Disease Control and Prevention. National, state, and urban area vaccination coverage among children aged 19-35 months - United States, 2005. MMWR Morb Mortal Wkly Rep. 2006;55:988-93.

11. Tau N, Yahav D, Shepshelovich D. Postmarketing safety of vaccin es approved by the U.S. Food and Drug Administration: a cohort study. Ann Intern Med. 2020;173:445-9. https://doi.org/10.7326/M20-2726

12. Centers for Disease Control and Prevention. Active Bacterial Core surveillance: surveillance reports. 2020 [cited $2020 \mathrm{Feb}$ 29]. https://www.cdc.gov/abcs/reports-findings/ surv-reports.html

13. Chapman R, Sutton K, Dillon-Murphy D, Patel S, Hilton B, Farkouh R, et al. Ten year public health impact of 13-valent pneumococcal conjugate vaccination in infants: a modelling analysis. Vaccine. 2020;38:7138-45. https://doi.org/10.1016/ j.vaccine.2020.08.068

14. Moher D, Liberati A, Tetzlaff J, Altman DG; PRISMA Group. Preferred reporting items for systematic reviews and meta-analyses: the PRISMA statement. PLoS Med. 2009; 6:e1000097. https:// doi.org/10.1371/journal.pmed.1000097

15. Loo JD, Conklin L, Fleming-Dutra KE, Deloria Knoll M, Park DE, Kirk J, et al. Systematic review of the effect of pneumococcal conjugate vaccine dosing schedules on prevention of pneumonia. Pediatr Infect Dis J. 2014;33:S14051. https://doi.org/10.1097/INF.0000000000000082

16. Tan TQ. Pediatric invasive pneumococcal disease in the United States in the era of pneumococcal conjugate vaccines. Clin Microbiol Rev. 2012;25:409-19. https:/ / doi.org/10.1128/CMR.00018-12

17. Tin Tin Htar M, van Den Biggelaar AHJ, Sings H, Ferreira G, Moffatt M, Hall-Murray C, et al. The impact of routine childhood immunization with higher-valent pneumococcal conjugate vaccines on antimicrobial-resistant pneumococcal diseases and carriage: a systematic literature review. Expert Rev Vaccines. 2019;18:1069-89. https:/ / doi.org/10.1080/147 60584.2019.1676155

18. Myint TTH, Madhava H, Balmer P, Christopoulou D, Attal S, Menegas D, et al. The impact of 7-valent pneumococcal conjugate vaccine on invasive pneumococcal disease: a literature review. Adv Ther. 2013;30:127-51. https:/ / doi.org/10.1007/s12325-013-0007-6

19. Aliberti S, Kaye KS. The changing microbiologic epidemiology of community-acquired pneumonia. Postgrad Med. 2013;125:31-42. https:/ / doi.org/10.3810/ pgm.2013.11.2710

20. Arguedas A, Soley C, Abdelnour A. Prevenar experience. Vaccine. 2011;29:C26-34. https://doi.org/10.1016/j.vaccine .2011.06.104

21. Balsells E, Guillot L, Nair H, Kyaw MH. Serotype distribution of Streptococcus pneumoniae causing invasive disease in children in the post-PCV era: a systematic review and meta-analysis. PLoS One. 2017;12:e0177113. https://doi.org/10.1371/journal.pone.0177113

22. United States Census Bureau. Population and housing unit estimates tables. 2020 [cited 2020 Mar 1]. https:/ / www. census.gov/programs-surveys/popest/data/tables.html

23. Zhou X, de Luise C, Gaffney M, Burt CW, Scott DA, Gatto N, et al. National impact of 13-valent pneumococcal conjugate vaccine on ambulatory care visits for otitis media in children under 5 years in the United States. Int J Pediatr
Otorhinolaryngol. 2019;119:96-102. https:/ / doi.org/10.1016/ j.ijporl.2019.01.023

24. Simonsen L, Taylor RJ, Young-Xu Y, Haber M, May L, Klugman KP. Impact of pneumococcal conjugate vaccination of infants on pneumonia and influenza hospitalization and mortality in all age groups in the United States. MBio. 2011;2:e00309-10. https:/ / doi.org/10.1128/ mBio.00309-10

25. Foote EM, Singleton RJ, Holman RC, Seeman SM, Steiner CA, Bartholomew M, et al. Lower respiratory tract infection hospitalizations among American Indian/ Alaska Native children and the general United States child population. Int J Circumpolar Health. 2015;74:29256. https://doi.org/10.3402/ijch.v74.29256

26. Grijalva CG, Griffin MR, Nuorti JP, Walter ND; Centers for Disease Control and Prevention. Pneumonia hospitalizations among young children before and after introduction of pneumococcal conjugate vaccine-United States, 1997-2006. MMWR Morb Mortal Wkly Rep. 2009;58:1-4

27. Lessa FC, Spiller MT, Soda E, Weinberger D, Griffin MR, Grijalva CG, et al. Impact of introduction of infant vaccination with 13 -valent pneumococcal conjugate vaccine (PCV13) on pneumonia and invasive pneumococcal disease (IPD) hospitalizations in the United States, 2005-2014. Presented at: 11th International Symposium on Pneumococci and Pneumococcal Diseases; 2018 Apr 15-19: Melbourne, Victoria, Australia.

28. Tong S, Amand C, Kieffer A, Kyaw MH. Trends in healthcare utilization and costs associated with pneumonia in the United States during 2008-2014. BMC Health Serv Res. 2018;18:715. https:// doi.org/10.1186/s12913-018-3529-4

29. Grijalva CG, Nuorti JP, Arbogast PG, Martin SW, Edwards KM, Griffin MR. Decline in pneumonia admissions after routine childhood immunisation with pneumococcal conjugate vaccine in the USA: a time-series analysis. Lancet. 2007;369:1179-86. https://doi.org/10.1016/ S0140-6736(07)60564-9

30. Black S, Shinefield H, Baxter R, Austrian R, Elvin L, Hansen J, et al. Impact of the use of heptavalent pneumococcal conjugate vaccine on disease epidemiology in children and adults. Vaccine. 2006;24:S79-S80. https://doi.org/10.1016/j.vaccine.2005.01.132

31. Yildirim I, Little BA, Finkelstein J, Lee G, Hanage WP, Shea K, et al; the Massachusetts Dept. of Public Health. Surveillance of pneumococcal colonization and invasive pneumococcal disease reveals shift in prevalent carriage serotypes in Massachusetts' children to relatively low invasiveness. Vaccine. 2017;35:4002-9. https:/ / doi. org/10.1016/ j.vaccine.2017.05.077

32. Marom T, Tan A, Wilkinson GS, Pierson KS, Freeman JL, Chonmaitree T. Trends in otitis media-related health care use in the United States, 2001-2011. JAMA Pediatr. 2014;168:6875. https://doi.org/10.1001/jamapediatrics.2013.3924

33. Tong S, Amand C, Kieffer A, Kyaw MH. Trends in healthcare utilization and costs associated with acute otitis media in the United States during 2008-2014. BMC Health Serv Res. 2018;18:318. https:/ / doi.org/10.1186/s12913-018-3139-1

34. Ray GT, Pelton SI, Klugman KP, Strutton DR, Moore MR. Cost-effectiveness of pneumococcal conjugate vaccine: an update after 7 years of use in the United States. Vaccine. 2009;27:6483-94. https:/ / doi.org/10.1016/ j.vaccine.2009.08.045

35. Pulido M, Sorvillo F. Declining invasive pneumococcal disease mortality in the United States, 1990-2005. Vaccine. 
2010;28:889-92. https://doi.org/10.1016/j.vaccine.2009. 10.121

36. World Health Organization. Estimated Hib and pneumococcal deaths for children under 5 years of age, 2000. 2014 [cited 2020 Mar 1]. https:/ / www.who.int/ immunization/monitoring_surveillance/burden/estimates/ Pneumo_hib/en

37. Shiri T, Khan K, Keaney K, Mukherjee G, McCarthy ND, Petrou S. Pneumococcal disease: a systematic review of health utilities, resource use, costs, and economic evaluations of interventions. Value Health. 2019;22:1329-44. https:/ /doi.org/10.1016/j.jval.2019.06.011

38. Dagan R, Ben-Shimol S, Greenberg D, Givon-Lavi N. A prospective, population-based study to determine the incidence and bacteriology of bacterial conjunctivitis in children $<2$ years following PCV7/PCV13 sequential implementation. Clin Infect Dis. 2020 Mar 4 [Epub ahead of print]. https://doi.org/10.1093/cid/ciaa197
39. Porat N, Benisty R, Givon-Lavi N, Trefler R, Dagan R. The impact of pneumococcal conjugate vaccines on carriage of and disease caused by Streptococcus pneumoniae serotypes 6C and 6D in southern Israel. Vaccine. 2016;34:2806-12. https://doi.org/10.1016/j.vaccine.2016.04.043

40. Jansen KU, Anderson AS. The role of vaccines in fighting antimicrobial resistance (AMR). Hum Vaccin Immunother. 2018;14:2142-9. https://doi.org/10.1080/21645515. 2018.1476814

41. Wantuch PL, Avci FY. Current status and future directions of invasive pneumococcal diseases and prophylactic approaches to control them. Hum Vaccin Immunother. 2018; 14:2303-9. https://doi.org/10.1080/21645515.2018.1470726

Address for correspondence: Matt Wasserman, Vaccines Outcomes \& Evidence, Pfizer Inc., 235 42nd Street, New York, NY 10017, USA; email: matt.wasserman@pfizer.com

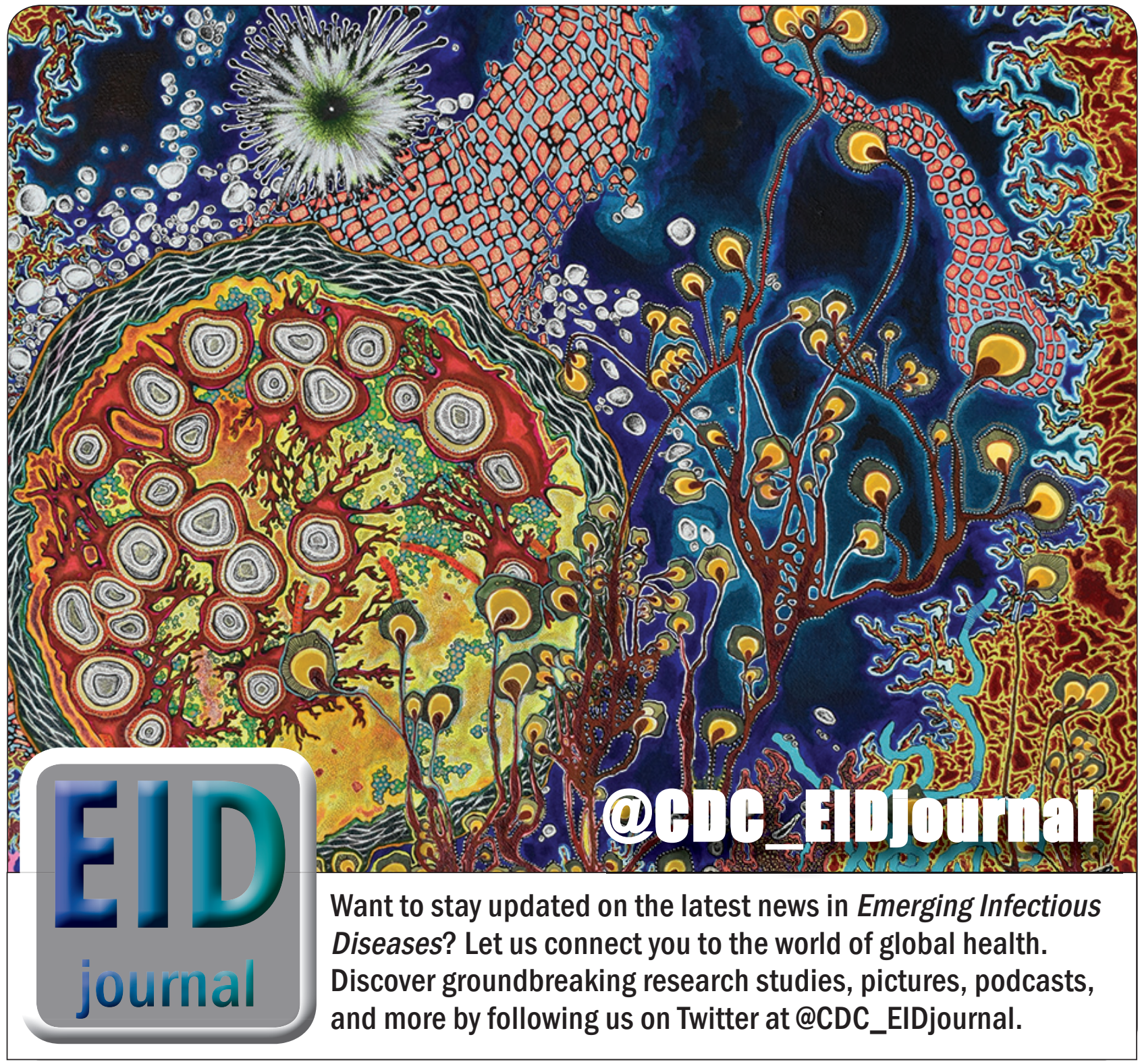

